\title{
An Extended Endochronic Theory Formulation of Plastically Deformed Damaged Indian Bamboo under Uniaxial Compression
}

Fozao DS ${ }^{1 *}$, Foudjet EA ${ }^{2}$, Fokwa $D^{3}$ and Kouam $A^{4}$

${ }^{1}$ Ministry of Public Works, Yaoundé, Cameroon

${ }^{2}$ Cresa Foret Bois, University of Dschang, Cameroon

${ }^{3}$ Department of Civil Engineering, ENSET, University of Douala, PO Box 1872, Douala, Cameroon

${ }^{4}$ ENSTP, Yaoundé, Cameroon

\begin{abstract}
This paper presents a series of uniaxial compressive loading tests carried out on cylindrical samples of Indian bamboo, together with a stress strain model. The bamboo species Oxytenantera abyssinica, from the Congo Basin forest is used for these tests. The non-linear behavior of Indian bamboo may be attributed to two distinct mechanisms, plasticity and damage. These two mechanisms are described by the endochronic theory of plasticity and continuum damage mechanics. A dissipative model, taken into account plasticity and damage through various parameters is proposed. The parameters of the model are deduced from monotonic and cyclic uniaxial compressive tests. The proposed stress-strain modelis compared to the tests results and it can be found that the model fits well with experimental results.
\end{abstract}

Keywords: Isotropic damage; Bamboo; Endochronic theory of plasticity; Continuum damaged mechanics; Plastic strain, Principle of equivalent strain

\section{Introduction}

Bamboo is a fast growing fibrous plant available in abundance on the earth. Their culms can grow to their full height of 3-30 m within a few months due to the expansion of individual internodes already present in the buds. It consists of cellulosic fibers embedded in lignin matrix cellulose. Fibres are aligned along the length of the tree. It is considered as a unidirectional composite, with a maximum tensile flexural strength and rigidity in that direction. Over 1200 species have been identified globally. Bamboo has a very long history with human beings. It has been used widely for household products and extended to industrial applications due to advances in processing technology and increased marked demand [1]. The aim of the present study is to understand the behavior of bamboo, Oxytenantera abyssinica, under compressive loading and a mathematical model will be proposed.

\section{Material and Methods}

The major objective of this research work is to propose a model to simulate the general stress strain behavior of Indian bamboo under uniaxial compressive loading. This model is produced from the extended endochronic theory (that is the endochronic theory of plasticity coupled with damage). The proposed models are initiated to provide flexibility of mathematical expression and the behavior of this material is very well described by this model.

\section{Material}

The experiments carried out were performed using the bamboo species, Oxytenantera abyssinica, from the Congo basin rain forest. The specimens were obtained from dry soil. After harvesting the bamboo culm, the growth buds were carefully trimmed off for each species. Bamboo is the most important non wood species that grows in most tropical and subtropical zones. It has been shown that bamboo is a superior alternate for manufactured wood composites. It is inexpensive, fast growing, easily available, and having comparable physical and mechanical properties to wood [2]. Bamboo is a composite material with long and parallel cellulose fibers in its structure. Its growth rate is very high with most of the growth occurring during the first year and growth ceasing by the fifth year [3]. The strength of bamboo increases with age. The maximum strength occurs at the age of about 3-4 years [3] and after this age, the strength begins to decrease. The major morphological characteristic of bamboo is divided into the rhizome and the culm. The rhizome or the subterranean stem is the underground part of the bamboo while the culm is the upper ground part that contains the wood material. The culm is straight, hollow and cylindrical in shape having nodes and internodes. The function of the nodes is to prevent buckling. In the internodes, the cells are strongly oriented axially with no radial cell elements; therefore the transversal interconnection is provided only by the nodes. The bamboo culm is made up of tiny countless fibers known as cellulose embedded in a lignin matrix $[2,4]$. The cellulose fibers run the length of the culm, carrying nutrients between the roots and the leaves [5]. The cellulose fibers act as the reinforcing steel in reinforced concrete or glass fibers in fiber reinforced plastics [6]. The lignin is the thermoplastic resin in that fills the spaces between the cellulose fibers. The distribution of the cellulose fibers increases from the inside to the outside [5,6]. Generally, the cellulose fiber is stronger than lignin. About $70 \%$ of the fibers in bamboo is cellulose and in most bamboo species, the fibers constitute about $60 \%$ on the outside and $10 \%$ on the inside [6]. Bamboo is an orthotropic material, having particular mechanical characteristics in the three directions; that is along the longitudinal, the transverse and the radial directions. Also, bamboo is a biological material; therefore the mechanical characteristics are affected by conditions such as soil conditions, species, age, environmental conditions and position of

*Corresponding author: Fozao DS, Ministry of Public Works Yaoundé, Cameroon, Tel: 237 70051870; E-mail: zaoden@hotmail.com

Received July 31, 2014; Accepted August 07, 2014; Published August 13, 2014

Citation: Fozao DS, Foudjet EA, Fokwa D, Kouam A (2014) An Extended Endochronic Theory Formulation of Plastically Deformed Damaged Indian Bamboo under Uniaxial Compression. J Appl Computat Math 3: 178 doi:10.4172/21689679.1000178

Copyright: $\odot 2014$ Fozao DS, et al. This is an open-access article distributed under the terms of the Creative Commons Attribution License, which permits unrestricted use, distribution, and reproduction in any medium, provided the original author and source are credited. 
the culm within the bamboo [5]. Bamboo is one of the oldest building materials used by mankind in the tropical and subtropical zones. The bamboo culms have been widely used in building applications such as flooring, ceilings, walls, windows, and roof trusses, structural materials for bridges and scaffoldings for the construction of high rising buildings. The geometrical characteristics of the material specimens used are found on Table 1 . The bamboo culms were divided into three portions, the lower, the middle and the upper parts. The specimens were randomly selected and used for the experiments. Round hollow culms were used. Some specimens had nodes within the culms while others were obtained from the internodes. The ends of the specimens were sanded to make them smooth. The green bamboo was left in the laboratory for five (5) months for seasoning. After air drying, some specimens were soaked in water for forty three (43) days before testing (Figure 1). The moisture contents of some of the samples used for experiments are found in Table 2.

\section{Methods (experimental program)}

The purpose of the experimental program in this paper is to investigate the behavior of Indian bamboo under monotonic uniaxial compressive loading. The stress strain curves obtained for various

\begin{tabular}{|c|c|c|c|c|c|}
\hline \multirow[t]{2}{*}{$\mathbf{N}^{\circ}$} & \multirow[t]{2}{*}{ Specimen } & \multicolumn{2}{|c|}{ Diameter (mm) } & \multirow{2}{*}{$\begin{array}{l}\text { Length } \\
(\mathrm{mm})\end{array}$} & \multirow[t]{2}{*}{ Remarks } \\
\hline & & $\begin{array}{l}\text { External } \\
\text { (d1) }\end{array}$ & $\begin{array}{l}\text { Internal } \\
\text { (d2) }\end{array}$ & & \\
\hline 01 & T1 Bottom without node (Figure 4) & 35.25 & 28.54 & 190 & \multirow{5}{*}{$\begin{array}{l}\text { Samples air } \\
\text { dried before } \\
\text { testing }\end{array}$} \\
\hline 02 & T4 Bottom with node (Figure 5) & 34.25 & 24.65 & 184 & \\
\hline 03 & T4 Middle without node (Figure 6) & 33.3 & 26.3 & 184 & \\
\hline 04 & T5 middle without node (Figure 7 ) & 36.45 & 27.6 & 184 & \\
\hline 05 & T6 Bottom without node (Figure 8) & 38.5 & 32.75 & 186 & \\
\hline 06 & T2 Bottom with node (Figure 9) & 39.5 & 19.5 & 188 & $\begin{array}{l}\text { Samples } \\
\text { soaked in } \\
\text { water for } 43 \\
\text { days before } \\
\text { testing. }\end{array}$ \\
\hline
\end{tabular}

Table 1: Geometrical characteristics of the Oxytenantera abyssinica specimens used

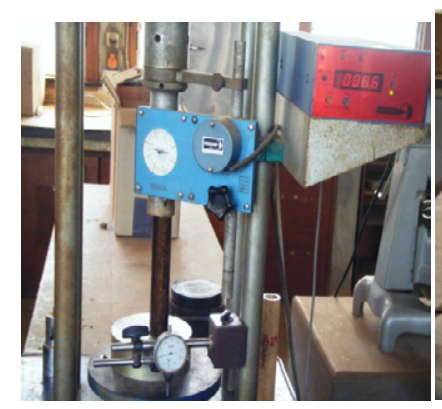

a) Specimen under the press

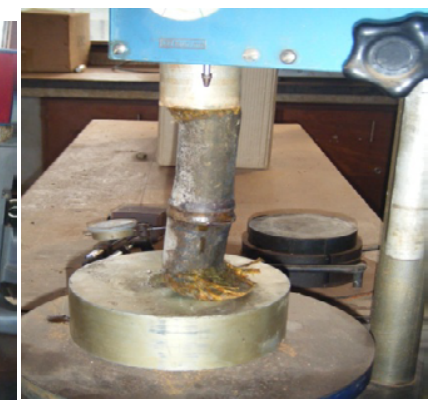

b) Wet sample under the press
Figure 1: (a) and (b): Specimens under the press for testing

\begin{tabular}{|c|c|c|}
\hline $\mathbf{N}^{\circ}$ & Specimen & Moisture content (\%) \\
\hline 01 & T1 Bottom & 16.81 \\
\hline 02 & T4 Bottom & 17.14 \\
\hline 03 & T4 Middle & 15.07 \\
\hline 04 & T5 Middle & 15.98 \\
\hline 05 & T6 Bottom & 16.42 \\
\hline 06 & T2 Bottom & 107.5 \\
\hline
\end{tabular}

Table 2: Moisture content.

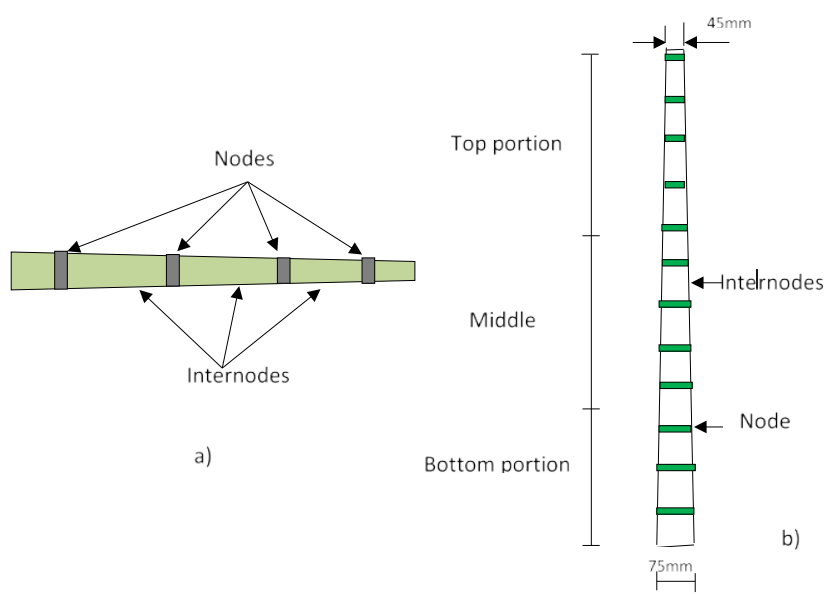

Figure 2: Various sections of the bamboo Culm.
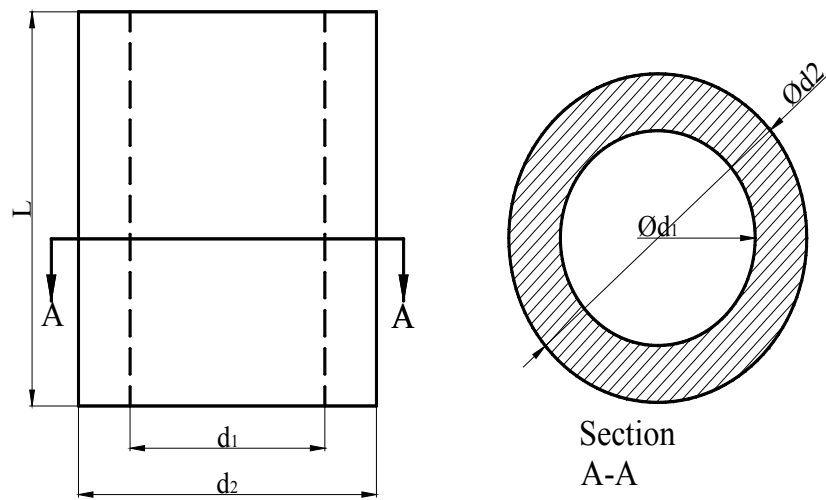

Figure 3: Typical bamboo culm modeled as a hollow cylinder.

specimens are presented and analyzed to calibrate the analytical model. To investigate the behavior of Indian bamboo under uniaxial compressive loading, two different loading regimes are employed as follows:

- Monotonic loading.

- Cycles to the envelope curves.

It is assumed in this study that the envelope curves are similar to the stress strain curves under monotonic uniaxial compressive loading. After cutting the specimens from the culm, the ends were sanded to make them smooth. Each specimen was placed in the testing machine and the compression load applied parallel to the grain. For the monotonic loading, the specimens were loaded continuously until they were completely damaged while for the cyclic compression loading, the specimens were loaded till the first crack and then unloaded to zero loads, then reloaded again. Reloading and unloading cycling was continued until the specimen was completely damaged. A $5000 \mathrm{kN}$ universal press in which is incorporated a digital micrometer, with adjustable speed is used. The deformations were monitored using the digital micrometer and loads were read after every $0.2 \mathrm{~mm}$ of deformation. Figure 2 shows the general setup. The stresses and strains were calculated using the original specimen dimensions and using the conventional expressions for these quantities. Figure 2 shows a general morphology of a bamboo culm. The bamboo culm was modeled as a hollow cylinder as shown on Figure 3. Stress-strain diagrams were plotted as shown on the diagrams 
Citation: Fozao DS, Foudjet EA, Fokwa D, Kouam A (2014) An Extended Endochronic Theory Formulation of Plastically Deformed Damaged Indian Bamboo under Uniaxial Compression. J Appl Computat Math 3: 178 doi:10.4172/2168-9679.1000178

from Figure 4. The stress strain diagrams shown in Figures 4-11 and used for this study were obtained from tests carried out on specimens of the bamboo species Oxytenantera abyssinica. In the literature, no models obtained from endochronic theory of plasticity coupled with damage are available for modeling the behavior of this material under monotonic uniaxial compressive loadings.

\section{Modeling of the monotonic uniaxial compression loading of} Indian bamboo

The curve shown in Figure 5 is a typical curve showing the results

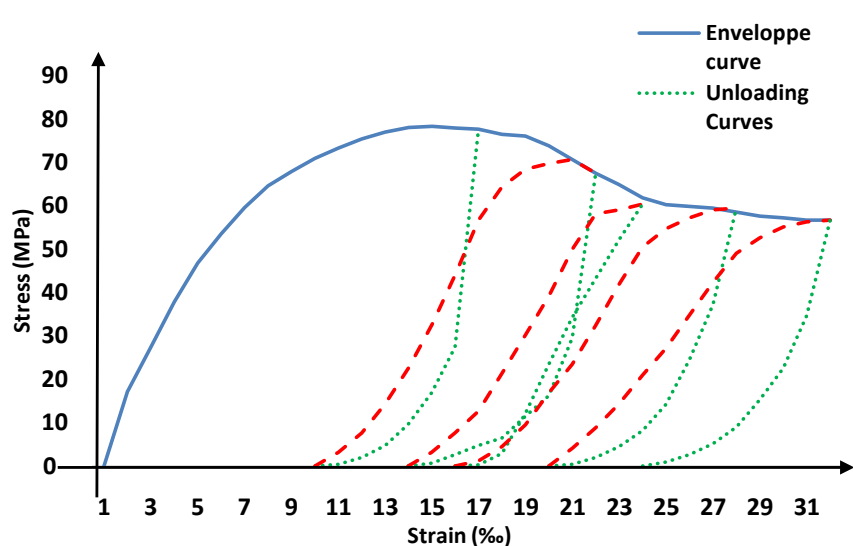

Figure 4: Typical stress-strain diagram (cyclic curves together with monotonic curve.

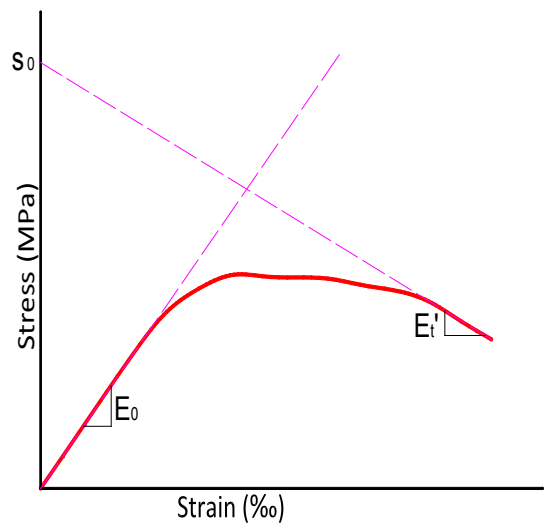

Figure 5: Indian Bamboo under monotonic compressive loading

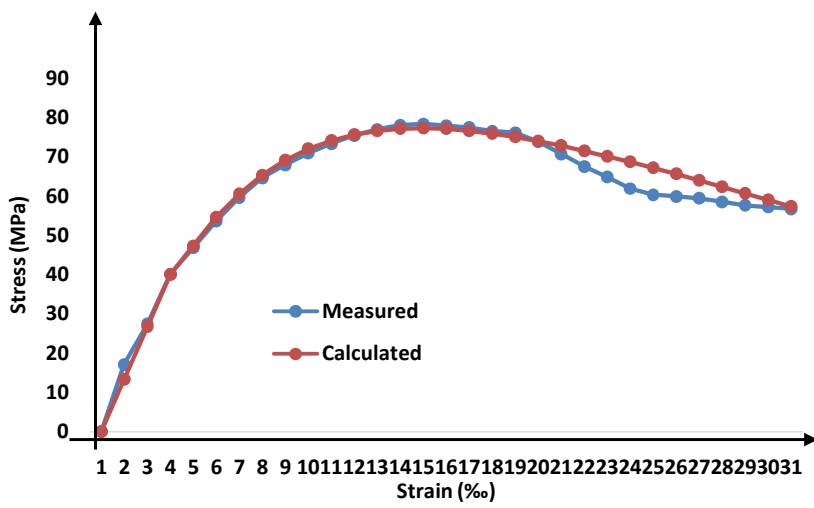

Figure 6: Measured and calculated data, for T1bottom without node.

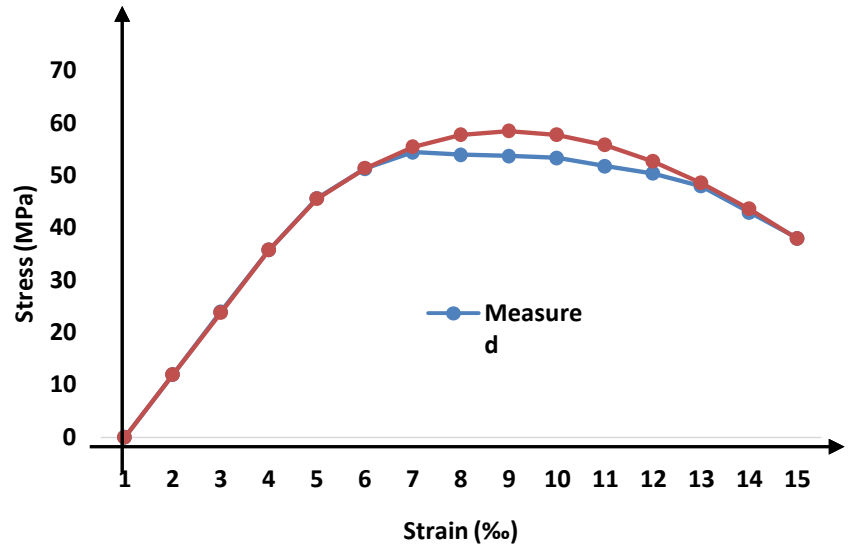

Figure 7: Measured and calculated data compared (T4 bottom with node).

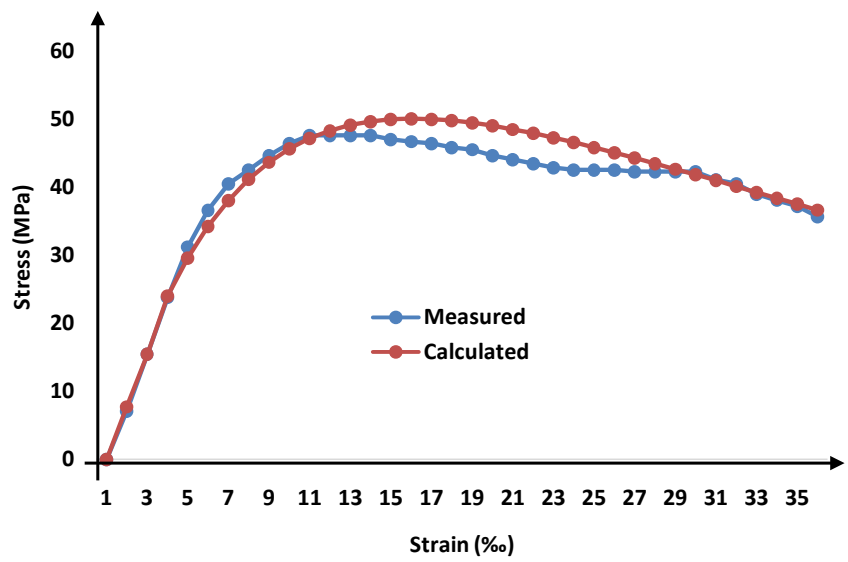

Figure 8: Measured and calculated data, for T4 middle without node.

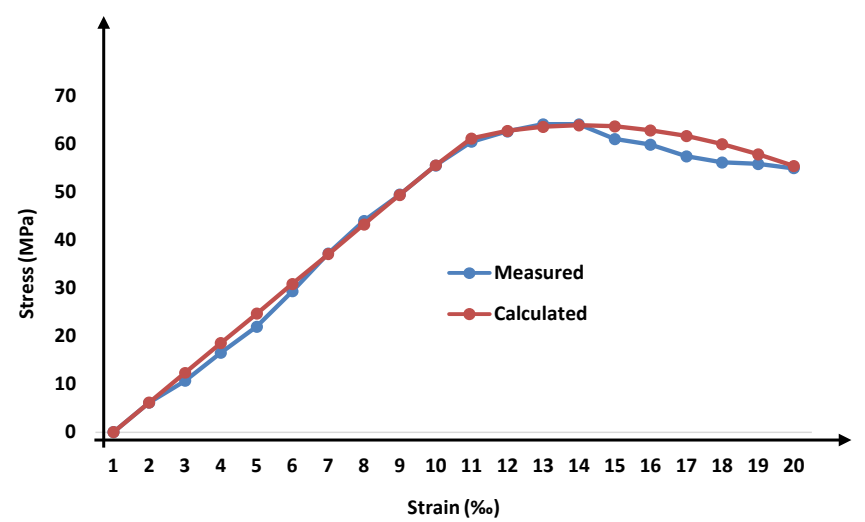

Figure 9: Measured and calculated data, for T5 middle without node.

of a cyclic uniaxial unloading and reloading test on a specimen of bamboo. It is used to explain the parameters that are used to predict the unloading and the reloading paths of the curves. Mathematical modeling of engineering materials is important in the design of engineering structures. The model should be able to explain, in the best way, the mechanical behavior of the material and should be as simple as possible to facilitate its use. The model produce in this article satisfies these requirements. In this section, analytical expressions for stress strain relations for bamboo subjected to monotonic uniaxial 
compression loading are developed. The models developed are based on constants which are functions of the strength of the bamboo species and they can be acquired from experimental results. The constants from the experimental stress strain diagrams that are used for this modeling are shown in Figure 5. The strain at the elastic limit, the modulus of elasticity, the slope of the stress strain curve at large strains as well as the intercept of the asymptotic curve at large strains are used. Figure 5 gives the definition of the various parameters used to predict the behavior of Indian bamboo under uniaxial monotonic compressive loading. These parameters are:

the modulus of elasticity $E_{0}$;

the value of the slope of the tangent curve at large strains $E_{t}^{\prime}$;

the intercept of the asymptote curve $\sigma_{0}$ at large values of $\varepsilon$ with the stress axis, represented on the diagram by $\mathrm{S}_{0}$;

the strain at yield $\varepsilon_{0}$;

Above the linear zone of the curve, the total strain can be considered to be made up of two parts, the elastic strain $\left(\varepsilon_{\mathrm{e}}\right)$ and the plastic strain $\left(\varepsilon_{\mathrm{p}}\right)$. The plastic strain is due to the internal modification of the structure of the material.

\section{Endochronic theory of plasticity}

The endochronic theory of plasticity was developed by Valanis in the early seventiesfrom the thermo-mechanical theory of internal variables [7-11]. The deformation history is represented by a monotonically increasing time-like parameter $\zeta$ known as the endochronic time [12]. This endochronic time that governs the rate independent evolution of stress and strain in materials $[9,10]$ also accounts for the intrinsic material properties [12]. This theory was later modified to introduce the effect of damage. In this paper, the endochronic theory of plasticity together with isotropic damage is used to produce a mathematical model that can be used to explain the behavior of Indian bamboo under uniaxial compression. The model is formulated within the framework of thermodynamics and implements a strong coupling between plasticity and damage. The constitutive equations for the damaged material are written according to the principle of strain equivalence between the virgin Indian bamboo material and the damaged material. In this approach the damaged Indian bamboo is modeled using the constitutive laws of the effective undamaged material, the nominal stresses being replaced by the effective stresses[13].

\section{Assumptions}

In the study of the behavior of Indian bamboo under uniaxial compression using the coupling between plasticity and damage, the following assumptions will be made:

- The material is considered to be isotropic;

- There is coupling between elasto-plasticity and damage;

- Deformations are considered to be small;

- Isothermal conditions are considered;

- The elastic strain vanishes when failure takes place;

- The total strain $(\varepsilon)$ can be decomposed into the elastic $\left(\varepsilon^{\mathrm{e}}\right)$ and plastic strains $\left(\varepsilon^{\mathrm{p}}\right)$.

The system to study is nonlinear and the principles of irreversible thermodynamics are applied. The first law of thermodynamics is widely used in the development of the internal variable theory. The system possesses an internal energy with an internal energy density. The change of the internal energy of the system may be accomplished through a purely thermal process or an adiabatic process. The thermal process will change the temperature of the system while the adiabatic process, through the work done will change the position of the boundary of the system, causing the system to deform. Therefore temperature and strain are measures of the internal energy of the system and are known as state variables since they serve to define the state of the system. The internal energy is one of the state functions that is used to represent the thermodynamic state of the system. The other state functions used are the specific entropy and the Helmholtz free energy. In irreversible systems, the state functions are not uniquely determined by the state variables strain and temperature. Other parameters, which are representative of the internal structure of the material are required. These parameters are called internal (or intrinsic) variables. It is assumed that sufficient additional state variables can always be found to describe the thermodynamic state of an irreversible system. These additional variables are known as internal variables. Therefore for an irreversible system, the state functions are functions of strain, temperature, and internal variables [12]. One of the original ideas of Valanis [7] was to develop a theory that describes the stress-strain curve without a yield surface. The stress-strain curve of Indian bamboo under uniaxial compression does not show any apparent yield point.

\section{Theoretical formulation of the simple endochronic theory}

In the simple endochronic theory, the total strain is used to measure the endochronic time. This theory is derived for small isothermal deformations. The endochronic time used in the theoretical formulation of the simple endochronic theory is given by:

$$
d \zeta=d \varepsilon
$$

The stress in the elastic plastic solid under uniaxial compression can be determined by using the convolution integral found in equation (2)[Han-Chin Wu (2005) and Watanabe]:

$$
\sigma=\int_{0}^{z} E\left(z-z^{\prime}\right) \frac{d \varepsilon}{d z^{\prime}} d z^{\prime}
$$

In the above expression, $\mathrm{d} \varepsilon$ is the total strain rate, $\mathrm{z}$ is the intrinsic time scale and $\mathrm{E}(\mathrm{z})$ is the kernel function. The intrinsic time scale can be determined from equation (3).

$$
d z=\frac{d \zeta}{f(\zeta)}
$$

In equation (3), $\zeta$ is the intrinsic (or the endochronic) time and $f(\zeta)$ is the scaling factor which is identified to the isotropic hardening or isotropic softening function. The case of $f(\zeta)=1$ corresponds to no isotropic hardening or softening. The function $f(\zeta)$ is a monotonically increasing function [12]. The kernel function may be given by the function in equation (4) [12]

$$
E(z)=E_{0} e^{-(\delta z)}
$$

where $\delta$ is a material constant and $E_{0}$ is Young's modulus of the material. The softening function can be given by equation(5) [12]:

$$
f(\zeta)=1+\beta \zeta
$$

where $\beta$ is the softening or hardening coefficient. Therefore we have:

$$
f(z)=\frac{d \zeta}{d z}=\frac{k d \varepsilon}{d z}=e^{\beta z}
$$


For materials with softening behaviour such as Indian bamboo, the coefficient $\beta$ is negative, (i.e. $\beta \leq 0$ ).

\section{Damage of materials}

Damage can be defined as the process of nucleation, growth and coalescence of voids in materials, processes that take place at a micro scale. These mechanisms will lead to a vanishing load carrying capacity of the material, as the damage density approaches unity. The accumulation of micro-damages has a tendency to form macroscopically localized damage, which is precursor to failure. This progressive physical process of degradation of the material mechanical properties will lead to damage and consequently leading the material to complete failure [13]. A material that is exposed to loading will at a certain total strain level starts to deteriorate and small cracks, voids and cavities of various forms will appear. The collective effect of these voids is called damage. Within the field of Continuum Damage Mechanics, internal variables are introduced to enable the material to be modeled as a continuum [14]. In Continuum Mechanics, damage is introduced as a state variable to model the effect of geometrical modification appearing within the material. An isotropic damage model, formulated within the thermodynamic framework, is considered. In Continuum Damage Mechanics, the constitutive equations for the damaged material can either be written according to the hypothesis of strain energy equivalence or the strain equivalence. In order to use the principle of continuum damage mechanics to derive these constitutive equations, use is made of a fictitious undamaged continuum, which is mechanically equivalent to the actual damaged continuum. In the fictitious undamaged configuration, all types of damages including both voids and cracks are removed from the element being studied.

\section{Principle of strain energy equivalence and strain equivalence}

Isotropic scalar damage theories are very often used in the study of Continuum Damage Mechanics. In these theories, use is made of the effective stress concept, where the stress is given as:

$$
\hat{\sigma}=\frac{\sigma}{1-D}
$$

In equation (7), $\hat{\sigma}$ is the effective stress applied on the fictitious undamaged bamboo material, $\sigma$ is the stress on the actually damaged material while $\mathrm{D}$ is the classical damaged variable introduced by Kachanov, defined as the ratio between the cross sectional area occupied by cracks together with voids and the total cross sectional area $[12,14]$.

\section{Strain energy equivalence principle}

The principle of strain energy equivalence introduced by Cordebois et al., [15] is widely used in Continuum Damage Mechanics to develop a class of damage representation theories [16]. This principle states that the elastic strain energy of the damaged material is the same in form as that of an undamaged material except that the stress and the strain are replaced by the effective stress and the effective strain $[12,13,16]$. The stored elastic energy W, in the damaged Indian bamboo material is given as:

$$
W=\frac{\sigma^{2}}{2 E}
$$

where $\mathrm{E}$ is the stiffness of the damaged material. The stored elastic energy in the fictitious undamaged Indian bamboo material is as:

$$
W=\frac{\hat{\sigma}^{2}}{2 E_{0}}
$$

where $E_{0}$ is the stiffness of the undamaged material. Using the principle of elastic energy equivalence and applying equation (7), the relationship between the stiffness of the actually damaged Indian bamboo material $\mathrm{E}$ and the stiffness of the undamaged material $\mathrm{E}_{0}$ can be given as:

$$
E=(1-D) E_{0}
$$

\section{Strain equivalence principle}

The principle of strain equivalence as postulated by Lemaitre is also widely used by some researchers to develop damaged theories in Continuum Damage Mechanics. The concept of strain equivalence relies on the assumption that there exists an undamaged fictitious material, whose response functions serve to establish the corresponding functions for the real damaged material by setting in relation the elastic strain quantities. As such the effective stress variables have to be used for the undamaged fictitious material. The principle of strain equivalence states that the strain associated with a damaged state under an applied stress is equivalent to the strain associated with its undamaged state under an effective stress. With this principle, the effective material behavior is represented in the effective stress and the actual strain space [16]. An equivalent energy state does not exist and effective strains are not employed [16]. The strain relation is given as follows:

$$
\varepsilon^{e}=\frac{\sigma}{E}=\frac{\hat{\sigma}}{E_{0}}
$$

Using this relation and equation (7), we have:

$$
E=(1-D) E_{0}
$$

From studies made by Olsson et al., [14] short comings exist for the strain and strain energy equivalence principles. These shortcomings are:

- The elastic strain will not be equal to zero when failure takes place;

- The damage rate at some point will decrease with increasing loading, and as a consequence, $\mathrm{D}$ will approach unity when the strain approaches infinity (i.e. $\mathrm{D}=1$ is an asymptotic value). In order to find a formulation for elasto-plasticity coupled with damage, that removes the shortcomings identified with the strain and energy equivalence postulates, the strain equivalence postulate can be modified by modifying the equation relating the stiffness of the actually damaged material and the stiffness of the undamaged material. Therefore the stiffness of the damaged material is given by introducing a material constant, $\mathrm{q}$, according to the works of Mattias Olsson et al., [14] as:

$$
E=E_{0}(1-q D)(13) \text { where } \mathrm{q} \text { is a material constant. }
$$

\section{Coupled damage/plasticity}

The non-linear behaviour of Indian bamboo may be attributed to plasticity and damage. These two degradation phenomena are described best by the theories of plasticity and continuum damage mechanics. Thus a multi-dissipative model that accounts for both plasticity and damage is necessary. This is accomplished by adapting two potential functions, one for plasticity and the other for damage. After the introduction of the standard endochronic theory by Valanis within the early 70s, many researchers have tried to modify it by the introducing a damage variable. Many models estimating the microdamage accumulation in materials have been published. Some of these models are based on damage micro-mechanics (known as micromechanical damage models) while others, based on the continuum damage theory, are known as phenomenological damage models [13]. 
Phenomenological models, which are applied in this article to explain the damage mechanics of Indian bamboo, are based on the initial concept of Kachanov (1958) who was the first to introduce the one dimensional damage variable for the isotropic case. The damage variable may be interpreted as the effective surface density of micro-damages per unit volume [13,16]. Kachanov pioneered the subject of continuum damage mechanics by introducing the concept of effective stress. Therefore the concept of effective stress is the foundation of Continuum Damage Mechanics [16]. This concept is based on considering a fictitious undamaged configuration of a body and comparing it with the actual damaged configuration. Quite often, materials undergo a strong plastic deformation, which has a major influence on the damage evolution and vice versa [13]. Many models have been introduced with coupling between damage and plasticity. In this study, it is assumed that there is coupling between plasticity and damage. Therefore, in carrying out the studies on the behavior of Indian bamboo under uniaxial compression, the principles of Continuum Damage Mechanics coupled with elastoplasticity will be adopted. Consequently,in this section, the endochronic theory coupled with isotropic damage is used to explain the nonlinear behavior of Indian bamboo subjected to uniaxial compression loading. The additive decomposition of total strain $(\varepsilon)$ into elastic $\left(\varepsilon^{e}\right)$ and plastic strains $\left(\varepsilon^{\mathrm{p}}\right)$ will be used. Consequently we have:

$$
\varepsilon=\varepsilon^{e}+\varepsilon^{p}
$$

In equation (14), the plastic strain also includes the strain induced by damage [14]. In this case, the damage portion of the strain $\left(\varepsilon^{\mathrm{d}}\right)$ is not explicitly identified. It is incorporated in the plastic portion of the strain.

\section{Formulation of the evolution laws for elasto-plasticity coupled with damage}

The formulation of the evolution laws for elasto-plasticity coupled with damage is based on two approaches as suggested by Olsson et al. [14]. It is either assumed that damage evolution takes place only during plastic loading as assumed by Lemaittre or that damage evolution can evolve independently of the plastic loading as assumed by Olsson et al., [14]. In this study, it is assumed that the plasticity process evolves in the effective continuum hence the effective stress is the essential mechanism by which theories of elasto-plasticity are coupled with damage theories. Consequently a proper effective stress relation is very necessary [16]. Using the simple endochronic theory, the effective stress on the fictitious continuum can be determined. This will be done by substituting equations (3), (4), (5) and (6) in equation (2) and simplifying to give the equation below [12].

$$
\begin{aligned}
& \hat{\sigma}=\frac{E_{0}}{\beta n}(1+\beta \varepsilon)\left[1-\frac{1}{(1+\beta \varepsilon)^{n}}\right] \\
& n=\left(1+\frac{\delta}{\beta}\right)
\end{aligned}
$$

In equation (15), $\varepsilon$ is the total strain and $\beta$ is the softening coefficient that can be determined asfollows.

The asymptote at large $\mathcal{E}$ is found from equation (15) to give:

$$
\sigma=\frac{E_{0}}{\beta n}(1+\beta \varepsilon)
$$

From the intercept of this asymptote with the stress axis (i.e. at $\varepsilon=0, \sigma=\sigma_{0}$ ), we have:

$$
\beta=\frac{E_{0}}{\sigma_{0} n}
$$

The expression of $\mathrm{n}$ can be determined from the slope of equation (15), $E_{t}^{\prime}$ at large values of $\mathcal{E}$. Therefore we have:

$$
n=\frac{E_{0}}{E_{t}}
$$

where $E_{t} \quad m E_{t}^{\prime}$

$\mathrm{m}$ is a material constant. It can be concluded that equation (15) may be used to model the nonlinear stress-strain relation of Indian bamboo under uniaxial compression. However, it must be realized that this model is not suitable to model the constitutive relation of Indian bamboo because micro-cracks exist on the Indian bamboo specimens before they are loaded. When the loading is small, the micro-cracks are stable and the Indian bamboo behayes essentially as a linear elastic material until a certain threshold strain ${ }^{\mathcal{E}_{m}}$ is reached when the material behavior becomes nonlinear. This threshgld strain can be assumed to be equal to the strain at the elastic limit $\mathcal{E}_{0}$. From equations (7), (13) and (15) the stress on the actually damaged material is given as:

$$
\sigma=(1-D) \hat{\sigma}=(1-D) \frac{E_{0}}{\beta n}(1+\beta \varepsilon)\left[1-\frac{1}{(1+\beta \varepsilon)^{n}}\right]
$$

Where $D=1-\alpha e^{-\gamma \varepsilon}$

Therefore

$$
\sigma=\frac{E_{0}}{\beta n}(1+\beta \varepsilon)\left[1-\frac{1}{(1+\beta \varepsilon)^{n}}\right] \alpha e^{-\gamma \varepsilon}
$$

Before the stress threshold, the material Indian bamboo behaves elastically but after the threshold, the material behavior becomes inelastic. Therefore the stress equation is given below as:

$$
\sigma=\left\{\begin{array}{cc}
E_{0} \varepsilon & \text { for } \varepsilon \leq \varepsilon_{0} \\
\frac{E_{0}}{\beta n}(1+\beta \varepsilon)\left[1-\frac{1}{(1+\beta \varepsilon)^{n}}\right]\left(\alpha e^{-\gamma \varepsilon}\right) & \text { for } \quad \varepsilon \geq \varepsilon_{0}
\end{array}\right.
$$

The value of $a$ can be determined by equating the two expressions in equation (24) at $\varepsilon=\varepsilon_{0}$. From this we have:

$$
\alpha=\frac{\beta n \varepsilon_{0}}{e^{-\gamma \varepsilon_{0}}}\left[\frac{\left(1+\beta \varepsilon_{0}\right)^{(n-1)}}{\left(\left(1-\beta \varepsilon_{0}\right)^{n}-1\right)}\right]
$$

Substituting equation (25) in the second expression of equation (24), we have:

$$
\sigma=A(1+\beta \varepsilon)\left[1-\frac{1}{(1+\beta \varepsilon)^{n}}\right] e^{-\gamma \varepsilon_{0}\left(k_{0}-1\right)}
$$

Where

$$
k_{0}=\frac{\varepsilon}{\varepsilon_{0}}
$$




$$
A=E_{0} \varepsilon_{0}\left[\frac{\left(1+\beta \varepsilon_{0}\right)^{(n-1)}}{\left(\left(1+\beta \varepsilon_{0}\right)^{n}-1\right)}\right]
$$

Several cylindrical specimens from the Indian bamboo species Oxytenantera abyssinica were tested either under the monotonic uniaxial compression testing regime or under the uniaxial cyclic compression regime. Stress strain curves were plotted. The graphs in Figures 8 and 9 are obtained from monotonic uniaxial compression tests while the graphs in Figures 6-8 and Figure 11 are obtained from the envelope curves obtained from the cyclic uniaxial compression tests. A mathematical expression, derived from the simple endochronic theory of plasticity and the principles of ContinuumDamage Mechanicshas been provided to explain the behavior of Indian bamboo subjected to monotonic uniaxial compression loading. Tests data and data obtained using this mathematical expression are compared. The results are shown on the diagrams from Figure 6-11. The parameters used in the mathematical expression for the six specimens modeled are represented in Table 3.

\section{Discussion}

\section{Comparison of test result and proposed models}

From the stress-strain diagrams plotted above, it can be seen that bamboo exhibits plastic behavior, since permanent or residual

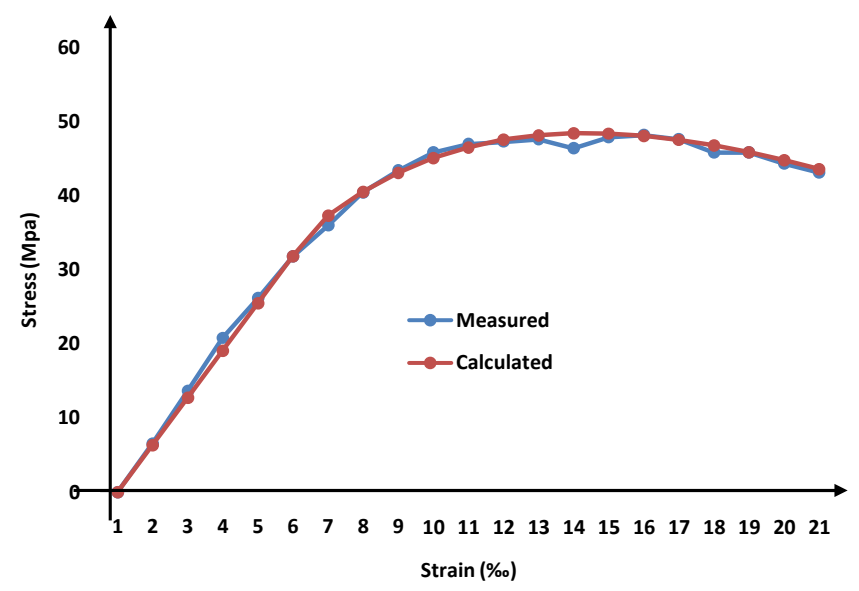

Figure 10: Measured and calculated data, for T6 bottom without node.

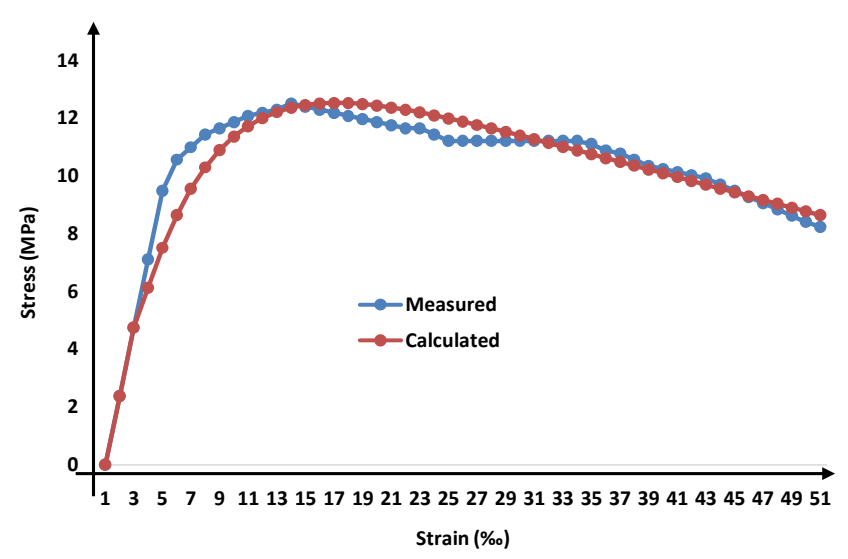

Figure 11: Measured and calculated data, for T2 bottom with node.

\begin{tabular}{|c|c|c|c|c|c|c|c|c|c|}
\hline $\begin{array}{l}\text { Parameter } \\
\text { Specimen }\end{array}$ & $\begin{array}{c}\mathrm{E}_{0} \\
(\mathrm{MPa})\end{array}$ & $\begin{array}{c}\varepsilon_{0} \\
\left(\mathbf{x} 10^{-3}\right)\end{array}$ & $\begin{array}{c}\mathrm{E}_{\mathrm{t}} \\
(\mathrm{MPa})\end{array}$ & $\alpha$ & $\begin{array}{c}\sigma_{0} \\
(\mathrm{MPa})\end{array}$ & m & $\mathbf{n}$ & $\beta$ & Y \\
\hline T1 Bo & & .20 & .21 & 0.96 & 0 & 2.35 & -9.47 & -14.42 & 0.5 \\
\hline T4 Bo & 77 & 3.26 & 52 & 1.05 & 108.58 & 1.12 & -2.13 & -47.45 & 0.85 \\
\hline T4 Middl & 349 & 2.11 & -633.14 & 1.12 & 52.08 & 0.56 & -11.61 & -12.16 & 0.85 \\
\hline T5 Middle & 677.8 & 9.78 & -231 & 1.06 & 3.5 & 2.75 & -2.45 & -27.74 & 3.5 \\
\hline T6 Bott & 6048.8 & 5.26 & -1130.62 & 1.05 & 55 & 1.0 & -5.35 & -20.56 & 0.85 \\
\hline T2 Bottom & 2231.41 & 2.13 & -108.43 & 0.94 & 13.7 & 0.58 & -20.58 & -7.91 & 0.6 \\
\hline
\end{tabular}

Table 3: Parameters used in the mathematical expression for the six specimens modeled.

strainremains when the loads are removed. Analytical expressions based on the endochronic theory of plasticity coupled with damage have been proposed to model the behavior of bamboo subjected to uniaxial compression loading. The proposed model is compared with experimental results. The figures shown above are used to compare the data obtained from the mathematical model to the experimental results. It is observed that the overall stress-strain behavior of the proposed model and tests results show similar configuration to each as well as fit very well with each other.

\section{Plasticity in bamboo}

It can be seen from the curves that after the proportional limits, the behavior of Indian bamboo deviates from the linear proportionality behavior and becomes nonlinear. Indian bamboo contains a large number of micro cracks even before any load has been applied. This property is very decisive for the mechanical behavior of bamboo. The micro cracks may be caused by thermal expansion and shrinkage during temperature fluctuations. The nonlinear behavior and the s-shape stress-strain curves of bamboo under uniaxial compressive stress can be associated with micro cracks propagation during load and stressinduced plastic flow in the specimen. Permanent residual strains are produced in the material after the proportional limit. These strains are not lost after the load is removed. The stress at which these permanent strains are produced is known as the yield stress. In this work, the yield stress is taken to be equal to the proportional limit of the material. The value of the yield stress for each specimen tested has been determined.

\section{Conclusion}

A constitutive model is proposed to predict the non-linear behavior of Indian bamboo under monotonic uniaxial compression loading. The experiments carried out were performed using the bamboo species, Oxytenantera abyssinica, from the Congo basin rain forest. The non-linear behavior of Indian bamboo may be attributed to two distinct mechanical processes, which are plasticity and damage. These two degradation phenomena are described best by the theories of plasticity and continuum damage mechanics. The model proposed in this article,is produced from the extended endochronic theory of plasticity (that is endochronic theory of plasticity coupled with isotropic damage). The test data from experimental investigations are compared to thismodel. The principle of equivalent strain has been used to produce the mathematical model. Two different loading regimes were employed to investigate thisnonlinear behavior of Indian bamboo under uniaxial monotonic compression loading. From the tests results, the major experimental parameters for the proposed analytical expression were obtained.

From the study, the following conclusions can be made

The nonlinear behavior of Indian bamboo can be modeled using the principles of endochronic theory of plasticity coupled with 
Citation: Fozao DS, Foudjet EA, Fokwa D, Kouam A (2014) An Extended Endochronic Theory Formulation of Plastically Deformed Damaged Indian Bamboo under Uniaxial Compression. J Appl Computat Math 3: 178 doi:10.4172/2168-9679.1000178

isotropic damage. Therefore Continuum Damage Mechanics principles are a powerful tool that can be used to analyze the behavior of Indian bamboo under uniaxial monotonic compression loading,

- When compared with the experimental results, the model shows satisfactory agreement with the experimental results;

- The asymptote curve as well as the slope of the tangent curve at large strains for the experimental curves are important elements that have been used in producing the model;

- All the input data required for the models are obtained from monotonic as well as cyclic compression tests results.

\section{Acknowledgement}

Many thanks go to Pr. Nkeng George Elambo, Director of the National Advanced School of Public Works Yaoundé, for permitting us to use the civil engineering laboratory of his school to carry out these experiments. Sincere thanks also go to Dr. Tchiengue Barthelemy for helping to identify the specimens, Mr. Thierry and Mr. Roland Forghap of the metallic workshops of the National Advanced Schools of Public Works Yaoundé and Buea Annex respectively for assisting in the preparation of the specimens not forgetting Koagne Tamba Chamberlin for assisting during the testing of the materials. I would also like to thank my beloved wife Mrs. Fozao Endaly Teckla Nwangum and my friend Mr. Taniform Peter for giving me the encouragementto carry out this work.

\section{References}

1. Fokwa D, Ngapgue F, Mpessa M, Tatietse TT (2012) Physical Characterization of two Cameroon bamboo species: Arundinaria alpina and Oxytenantera abyssinica. International Journal of Engineering and Technology 4: 82-92.

2. Chaowana $P$ (2013) Bamboo, An alternative raw material for wood and wood based composites. Journal of Material Science Research 2: 90-102.

3. Amada S, Untao S (2001) Fracture properties of bamboo composites. Composites: Part B 32: 451-459.

4. Janseen JJA (2000) Designing and building with bamboo. Technical University of Eindhoven, the Netherlands, Technical Report No 20, International Network of Bamboo and Rattan.
5. Naik NK (2004) Mechanical and Physico-Chemical properties of bamboo carried out by Aerospace Engineering Department, Indian Institute of Technology Bombay, Technical report.

6. Janssen JJA (1985) The Mechanical Properties of Bamboo. Technical University of Eindhoven PostPus. 513, 5600-Eindhoven, Netherlands Proceedings of International Bamboo Workshop, China 250-256.

7. Valanis KC (1970) A theory of viscoplasticity without a yield surface. Part I: general theory, 1970, report university of OWO USA.

8. Valanis KC (1971) A theory of viscoplasticity without a yield surface. Part II: application to mechanics of materials, 1971, 1970, report university of OWO USA.

9. Erlicher S, Point N (2008) Endochronic theory, non-kinematic hardening rule and generalized plasticity: a new interpretation based on generalized normality assumption. International Journal of Solids and Structures 43: 4175-4200.

10. Erlicher S, Nelly Point N (2008) Pseudo-potentials and loading surfaces for an endochronic plasticity theory with isotropic damage Journal of Engineering Mechanics.

11. Jain SK (2000) Advancement of endochronic theory in Engineering Mechanics, Europe Congress on Computational Methods in applied sciences and engineering. Barcelona 11-14 September 2000.

12. Han-Chin Wu (2005) Continuum Mechanics and Plasticity. Chapman and Hall/ CRC Press Company.

13. Abu Al-Rub RK, Voyiadjis GZ (2003) On the coupling of anisotropic damage and plasticity models for ductile materials. International Journal of Solids and Structures 40: 2611-2643.

14. Olsson M, Ristinmaa M (2005) A Physically Motivated Modification of the Strain Equivalence Approach. International Journal of Damage Mechanics 14: 25-50.

15. Cordebois JP, Sidoroff F (1979) Damage induced elastic anisotropy Mechanical Behavior of Anisotropic Solids/Comportment Méchanique des Solides Anisotropes 761-774.

16. Hansen NR, Schreyer HL (1994) A Thermodynamically Consistent Framework for Theories of Elasto-Plasticity Coupled with Damage. International Journal of Solids and Structures 31: 359-389. 